\title{
Vibrating String with a Variable Length
}

\author{
Abdelkader Megueni ${ }^{\dagger}$ \\ Department of Mechanics, Faculty of Engineering, University of Sidi-Bel-Abbes, (22000), Algeria
}

Lakhdar Gaffour
Institute of Physics, University of Sidi-Bel-Abbes, (22000), Algeria

(Received 28 November 2000; accepted 20 October 2003)

\begin{abstract}
The propagation of waves in a string with stationary boundary conditions is well known. The transverse vibrations of a string where one extremity is moving with a prescribed motion is considered. We cannot apply the method of separation of variables. This method leads to very complex equations. A solution developed previously by L. Gaffour is introduced in this study. ${ }^{1}$ The solution for the case of a string with a time-varying length with appropriate boundary conditions is examined. This solution is exact and is expressed in terms of functional Fourier series. It is developed for the linear motion of the extremity of the string. The modes of vibrations and the energy ratio are formulated in a precise way. The curves of eigenmodes, energy ratio, and energy are presented.
\end{abstract}

${ }^{\dagger}$ Member of the International Institute of Acoustics and Vibration (IIAV)

\section{INTRODUCTION}

The propagation of waves in a string for the case of timevarying length is a problem of one-dimensional wave propagation with moving boundary conditions. Confronted with the difficulty of finding an exact analytical method in order to solve such a problem, scientists and engineers have relied on numerical and approximate analytical methods. For simple cases, there are specific methods. ${ }^{\mathbf{5}, 6}$ However, more general mathematical methods are used for analytically solving such problems. These methods lead to the solutions of the functional or integro-differential equations. ${ }^{7-11}$ The method of separation of variables used by Tadashi Kotera leads to a complex solution. ${ }^{4}$ Such solutions are very cumbersome and complex. Recently, Y. M. Ram and J. Caldwell introduced a new method - the method of distorted images. ${ }^{3}$ This method remains limited and does not take into account the energy of the system. However, these methods do not allow the deduction and the understanding of the physical phenomena. More recently, Stefan Kaczmarczyk and Wieslaw Ostachowicz used the method of multiple scales to study the longitudinal dynamics of hoisting cables and to predict the responses near the resonance region. ${ }^{2}$ Nicolas Gonzales studied the stability of the energy of the wave equation for periodic motion. ${ }^{13}$ The method used in this paper is an exact analytical method based on analytical functions and conformal transformations. ${ }^{\mathbf{1 1 2}}$ It has been developed for solving a large class of systems with non-stationary dimensions. The main advantage of this method is that it gives the exact solution for all motions (parabolic, hyperbolic, etc.). The other advantages are the easy application, the exact formulation of the energy ratio, and the possibility of deducing the energy. By means of the method cited above, we have determined the exact solution of our problem, the modes, the energy ratio, the energy and discussed the results obtained.

\section{SUMMARY OF L. GAFFOUR METHOD}

\subsection{Principle of the Method}

The method consists of transforming the domain $B$ defined by:

$$
B=\{(\tau, x) / \tau \geq 0 ; 0 \leq x \leq l(\tau)\}
$$

into a band domain $B^{\prime}$ defined as:

$$
B^{\prime}=\left\{(\xi, \eta) / 0 \leq \eta \leq \eta_{0}\right\}
$$

where $\eta_{0}$ is fixed (Fig. 1), using a conformal transformation $F$ such that:

$$
F(w)=\tilde{f}(\xi, \tilde{\eta})+i \widetilde{g}(\xi, \tilde{\eta})
$$

and by imposing a condition upon $F{ }^{1}$ New real transformations are determined, which leaves the wave equation invariant in the fixed domain $B^{\prime}$, where the solution is well known.

The solution in the initial domain $B$ is obtained by using the inverse transform of $F$ noted $\psi$ such that $\psi(Z)=F^{-1}(W)$.

\subsection{Solution of the Problem}

Let us consider the wave equation:

$$
\frac{\partial^{2} u}{\partial x^{2}}-\frac{\partial^{2} u}{\partial \tau^{2}}=0
$$

with the symmetric initial conditions:

$$
\frac{\partial u}{\partial \tau}(x, 0)=\varphi_{1}(x) ; \quad \frac{\partial u}{\partial x}(x, 0)=\varphi_{2}(x)
$$

